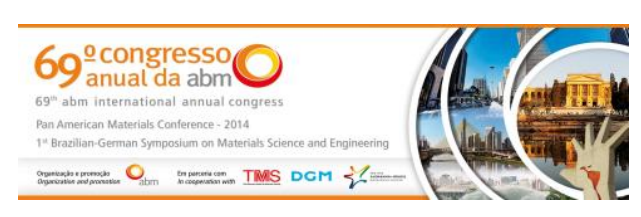

Tema: Iniciação Científico-tecnológica

\title{
MODELAGEM FÍSICA DA REMOÇÃO DE INCLUSÕES NO DISTRIBUIDOR DE LINGOTAMENTO CONTÍNUO*
}

\author{
Elisa Araujo Santos Darsie ${ }^{1}$ \\ Paula Rodrigues Caumo 1 \\ Felipe das Dores Machado² \\ Magda Beatriz Galant François ${ }^{3}$ \\ Antônio Cezar Faria Vilela ${ }^{4}$
}

\section{Resumo}

A busca na melhoria da qualidade dos aços, principalmente no caso de aços limpos, fez com que o distribuidor de lingotamento continuo também seja empregado para a remoção das inclusões responsáveis por prejudicar as propriedades mecânicas dos aços. Uma melhor eficiência desta função do distribuidor pode ser atingida com o conhecimento das características do escoamento do banho metálico de modo que se possam propor modificações na configuração do distribuidor. Esses fenômenos de escoamento e remoção de inclusões podem ser simulados mediante a utilização da modelagem física. O objetivo deste trabalho é avaliar quatro configurações internas de distribuidor por meio da qualificação do escoamento via ensaio de linhas de fluxo e quantificação de inclusões passantes pelo veio com o ensaio de remoção de inclusões. As variáveis de controle utilizadas neste estudo são: velocidade de lingotamento, presença de barreiras e presença de tubo longo. Os resultados apresentados para o distribuidor em questão mostram que para os casos com o tubo longo, o uso da barreira torna o escoamento predominantemente ascendente eliminando o curto-circuito e favorável a remoção de inclusões passando de 29,76 para $24,29 \%$ de inclusões passantes pelo veio. Enquanto que para os casos sem tubo longo, o uso da barreira proposta diminuiu o contato do aço com a escória sobrenadante, aumentou o curto-circuito e aumentou também o percentual de inclusões passantes pelo veio de 2,56 para 3,01\%.

Palavras-chave: Modelagem física; Remoção de inclusões; Distribuidor.

\section{PHYSICAL MODEL OF INCLUSION REMOVAL IN CONTINUOUS CASTING TUNDISH Abstract}

The search for improve the steel quality, mainly in the case of clean steel, is the reason to the tundish also to be employed to inclusion removal. These inclusions can strongly damage the mechanical proprieties of steel. The effectiveness of this tundish function demands the knowledge of the flow characteristics of metallic bath and it can propose modifications on the tundish configuration. This knowledge can be acquired by using the physical modeling to simulate the involved phenomenon. The objective of this work is to evaluate four internal configurations of tundish using the physical model of the fluid flow characterization and inclusion removal. The variables used in this study are speed of ingoting, presence of dams and presence of submerged inlet shroud. The results presented to the tundish used in this work show that, in case of the use of submerged inlet shroud, associated with the use of dams, the fluid flow becomes predominantly upflow eliminating the bypass flow and it is favorable to removal of inclusions, decreasing from 29.76 to $24.29 \%$ of the inclusions nonfloated. However, for the cases without submerged inlet shroud, the presence of dams decreased the contact of the steel with the slag, increasing the short circuit and increasing the percentage of the inclusions non-floated from 2.56 to $3.01 \%$.

Keywords: Tundish; Inclusion removal; Physical Model.

\author{
Graduando de Eng. Metalúrgica, LaSid, DEMET, EE, UFRGS, Porto Alegre, RS, Brasil. \\ Eng. Químico, Mestrando, LaSid, PPGE3M, EE, UFRGS, Porto Alegre, RS, Brasil. \\ Dr. INPL, Pesquisadora LaSid, UFRGS, Prof. Ulbra, Porto Alegre, RS, Brasil. \\ Prof. Dr.-Ing., LaSid, DEMET, PPGE3M, EE, UFRGS, Porto Alegre, RS, Brasil.
}

\footnotetext{
* Contribuição técnica ao 69 Congresso Anual da ABM - Internacional e ao $14^{\circ}$ ENEMET - Encontro Nacional de Estudantes de Engenharia Metalúrgica, de Materiais e de Minas, 21 a 25 de julho de 2014, São Paulo, SP, Brasil.
} 




\section{INTRODUÇÃO}

Com a crescente busca do setor industrial por aços com melhores características mecânicas, o processo de produção de aços tem seguido padrões necessários para que se possa obter um controle rígido quanto à quantidade, tamanho e morfologia de inclusões. A modificação do escoamento interno do distribuidor de lingotamento contínuo favorecendo a flotação das inclusões é uma maneira bastante eficaz no controle de limpeza do aço, como mostram alguns trabalhos [1-4].

O distribuidor é um dos equipamentos que torna possível o processo de lingotamento da fabricação de aços de modo contínuo. Ele é o responsável pela alimentação de aço líquido para os moldes e também pode atuar como pulmão durante a troca de panelas aumentando a produtividade do processo. Com o objetivo de melhorar a qualidade dos aços, especialmente no caso de aços "limpos" (clean steels), este equipamento também é empregado como um reator metalúrgico capaz de atuar na limpeza das inclusões do aço. Esta função é importante, pois as inclusões podem atuar como concentradoras de tensões e, consequentemente, diminuir as propriedades mecânicas dos aços. Convém ressaltar que o distribuidor é a última etapa do processo de lingotamento contínuo com volume e tempo de residência suficiente para as inclusões flotarem e serem retiradas do aço.

As inclusões não metálicas são intrínsecas ao processo de fabricação do aço. Podem ser originadas em diferentes pontos da aciaria, como por exemplo: a reoxidação do metal líquido por meio da exposição ao ar atmosférico, o processo de desoxidação (adição de alumínio e outros), o fenômeno de arraste de escória da panela, a emulsificação de escória no distribuidor e vários outros que podem ser encontrados na literatura [5].

O estudo do escoamento interno do distribuidor e o fenômeno de remoção de inclusões são essenciais para que se consiga reduzir o nível de inclusões não metálicas no aço. Uma das alternativas que podem ser utilizadas são os estudos sobre o distribuidor via modelagem física com a construção do distribuidor em acrílico em escala real (1:1) ou reduzida e que utilizam a água à temperatura ambiente como fluido simulador do aço líquido. Esses ensaios respeitam critérios de modelagem física (similaridade), como: as similaridades de Reynolds e Froude.

O objetivo deste trabalho é quantificar a remoção de inclusões para diferentes condições de escoamento no distribuidor envolvendo presença de barreiras, tubo longo e variação de velocidade de lingotamento. Paralelamente, o ensaio de linhas de fluxo é realizado com a motivação de fornecer suporte ao entendimento dos resultados obtidos no ensaio de remoção de inclusões.

\section{METODOLOGIA}

Para a realização dos ensaios foi utilizado um modelo físico construído em acrílico e pertencente ao Laboratório de Siderurgia da UFRGS. Este modelo é igual a um distribuidor real instalado em uma usina siderúrgico (escala real - 1:1). Nesses ensaios foram testados quatro tipos de configurações: sem o tubo longo e sem barreiras; sem tubo longo e com barreiras; com tubo longo e sem barreiras; com tubo longo e com barreiras. Além de testar a variação da velocidade de lingotamento na remoção de inclusões para as velocidades: $1,8 \mathrm{~m} / \mathrm{min} ; 2,2 \mathrm{~m} / \mathrm{min} ; 2,6 \mathrm{~m} / \mathrm{min}$. As medidas do distribuidor, das barreiras e tubo longo estão relatadas em Machado [6].

\footnotetext{
* Contribuição técnica ao 69 Congresso Anual da ABM - Internacional e ao $14^{\circ}$ ENEMET - Encontro Nacional de Estudantes de Engenharia Metalúrgica, de Materiais e de Minas, 21 a 25 de julho de 2014, São Paulo, SP, Brasil.
} 

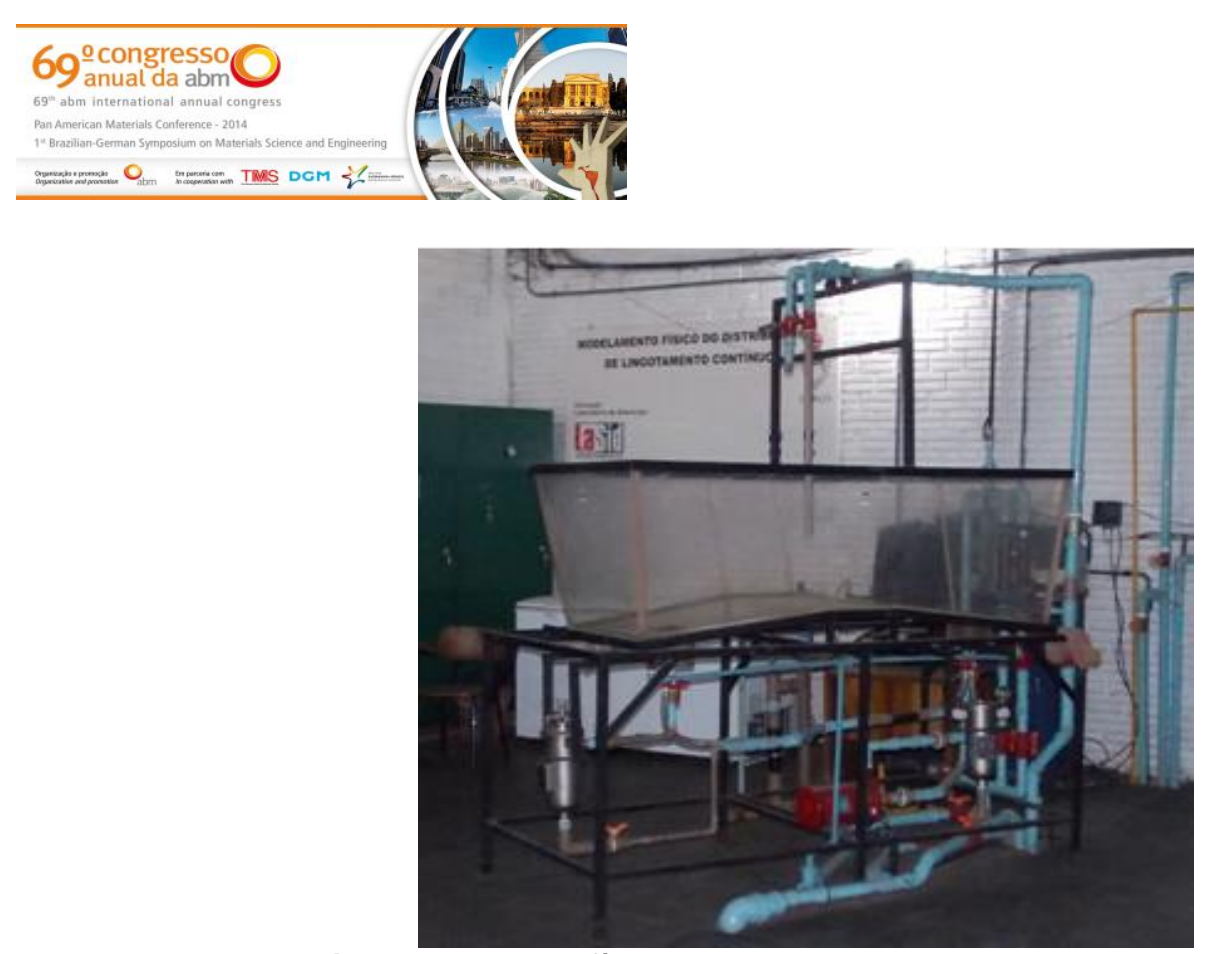

Figura 1. Modelo físico do distribuidor de lingotamento contínuo

A simulação física do aço líquido utilizando a água à temperatura ambiente é com base na viscosidade cinemática da água a $25^{\circ} \mathrm{C}$ que é similar à do aço na temperatura de processamento, aproximadamente $1600^{\circ} \mathrm{C}$, com isso respeita-se a similaridade de Reynolds para modelos em escala 1:1 [7]. Para simular a remoção de inclusões no distribuidor é necessário que o escoamento e os fenômenos de remoção de inclusões sejam similares à situação real. Certamente não é possível manter todas as condições idênticas à situação real. No entanto, é possível manter alguns critérios de similaridade que proporcionem resultados satisfatórios, como por exemplo, os critérios de similaridade geométrica, cinemática e dinâmica [8].

Como metodologia principal, os ensaios de linhas de fluxo tem o objetivo de identificar qualitativamente o escoamento do distribuidor sob o ponto de vista da remoção de inclusões. Desse modo, as linhas de fluxo fornecem informações para o entendimento dos resultados do ensaio de remoção de inclusões.

\subsection{Ensaio de linhas de fluxo}

O ensaio tem como objetivo visualizar as linhas de fluxo que percorrem dentro do distribuidor até que atinjam os veios. Com isso determinam-se áreas onde o líquido demora mais tempo para se misturar (zonas mortas) ou onde ele passa mais rapidamente, por exemplo, o curto-circuito. Na prática, as zonas mortas podem provocar maior perda de temperatura no aço e provocar heterogeneidade térmica na alimentação do molde. Do mesmo modo que o curto-circuito pode elevar a temperatura do molde.

O ensaio de linhas de fluxo é operado com o distribuidor em regime permanente e devido este ensaio ser preferencialmente qualitativo a velocidade de lingotamento utilizada é a intermediária $(2,2 \mathrm{~m} / \mathrm{min})$. Para a visualização do escoamento é injetado um pulso (3 segundos) de uma solução de flutuação neutra de permanganato de potássio $\left(\mathrm{KMnO}_{4}\right)$ de aproximadamente $400 \mathrm{ml}$ de volume. A injeção ocorre após o período de estabilização do escoamento (10 minutos) para que não haja interferências ao experimento. O caminho em que o corante percorre no modelo é acompanhado por meio de uma câmera filmadora, posicionada à frente do modelo para captura da vista frontal, conforme mostrado na Figura 2.

\footnotetext{
* Contribuição técnica ao 69 Congresso Anual da ABM - Internacional e ao $14^{\circ}$ ENEMET - Encontro Nacional de Estudantes de Engenharia Metalúrgica, de Materiais e de Minas, 21 a 25 de julho de 2014, São Paulo, SP, Brasil.
} 

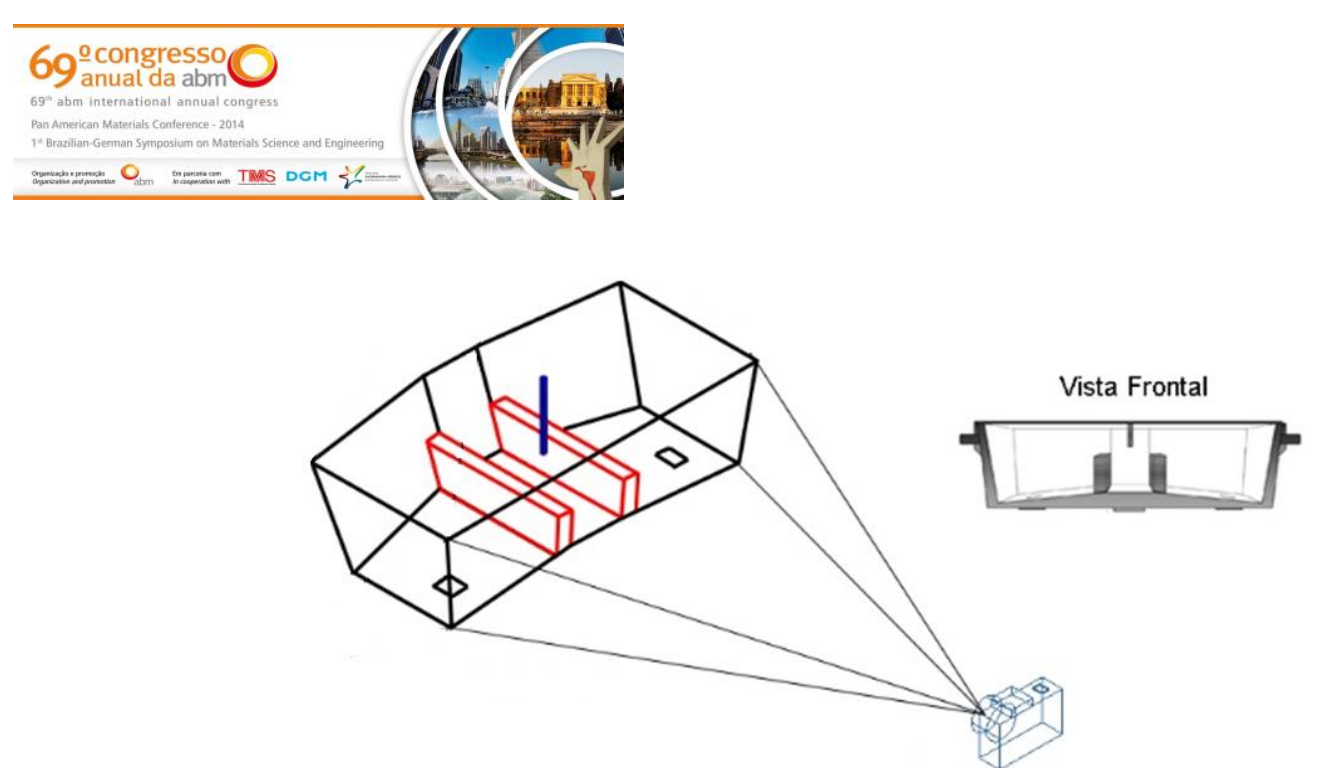

Figura 2. Posicionamento da câmera da vista frontal utilizada no ensaio de linhas de fluxo.

\subsection{Ensaio de remoções de inclusões}

O ensaio de remoção de inclusões é realizado com o distribuidor mantido em regime permanente e após a estabilização do escoamento (10 $\mathrm{min})$ é realizada a injeção das partículas de simulação de inclusões alumina na forma de pulso (3 segundos). Para formar uma mistura homogênea capaz de ser injetada no distribuidor é realizada a mistura de uma amostra de polietileno previamente homogeneizada (30 g) a uma solução de álcool etílico absoluto. O volume total da mistura é igual a $150 \mathrm{ml}$. Logo que a mistura é injetada, as micropartículas tendem a flotar em direção a superfície do distribuidor. Entretanto, uma quantidade de partículas passa pelo distribuidor e se direciona diretamente aos veios de saída, devido ao arraste gerado pela velocidade do escoamento. Para isso, há uma peneira metálica adaptada na saída do modelo que captura as partículas passantes pelo veio permitindo a sua quantificação. Após dois tempos de residência teórica, as inclusões capturadas no filtro são recolhidas, secadas em estufa para remover toda a umidade e pesadas. Desta maneira, é possível quantificar a porcentagem de inclusões (Equação 1) que saiu pelos veios e determinar quais configurações do distribuidor foram as mais favoráveis para a remoção de inclusões. Todos os ensaios são realizados em duplicata.

$\eta_{r}(\%)=\left(\frac{m_{c}}{m_{i}}\right) \times 100$

\section{RESULTADOS E DISCUSSÃO}

\subsection{Ensaio de linhas de fluxo}

Nas Figuras de 3 a 6, o tempo é mostrado na forma de escalas de cinza. Quanto mais claro o tom de cinza, maior é o tempo de ensaio após a injeção do corante. A as flechas amarelas indicam os fluxos preferenciais e os círculos pontilhados em vermelho indicam as zonas mortas.

\subsubsection{Distribuidor sem tubo longo e sem barreiras}

Para a configuração sem tubo longo e sem barreiras (Figura 3), o impacto da interface ar/água pode causar uma quebra dos vetores de velocidade, com isso a penetração do jato não é profunda. Todavia, essa configuração ocasiona um movimento ascendente ao escoamento, permitindo assim maior contato do aço com a escória, para só então se dirigir aos veios. Entretanto, o escoamento se divide em

* Contribuição técnica ao 69 Congresso Anual da ABM - Internacional e ao 14ํㅡㄹ ENEMET - Encontro Nacional de Estudantes de Engenharia Metalúrgica, de Materiais e de Minas, 21 a 25 de julho de 2014, São Paulo, SP, Brasil. 

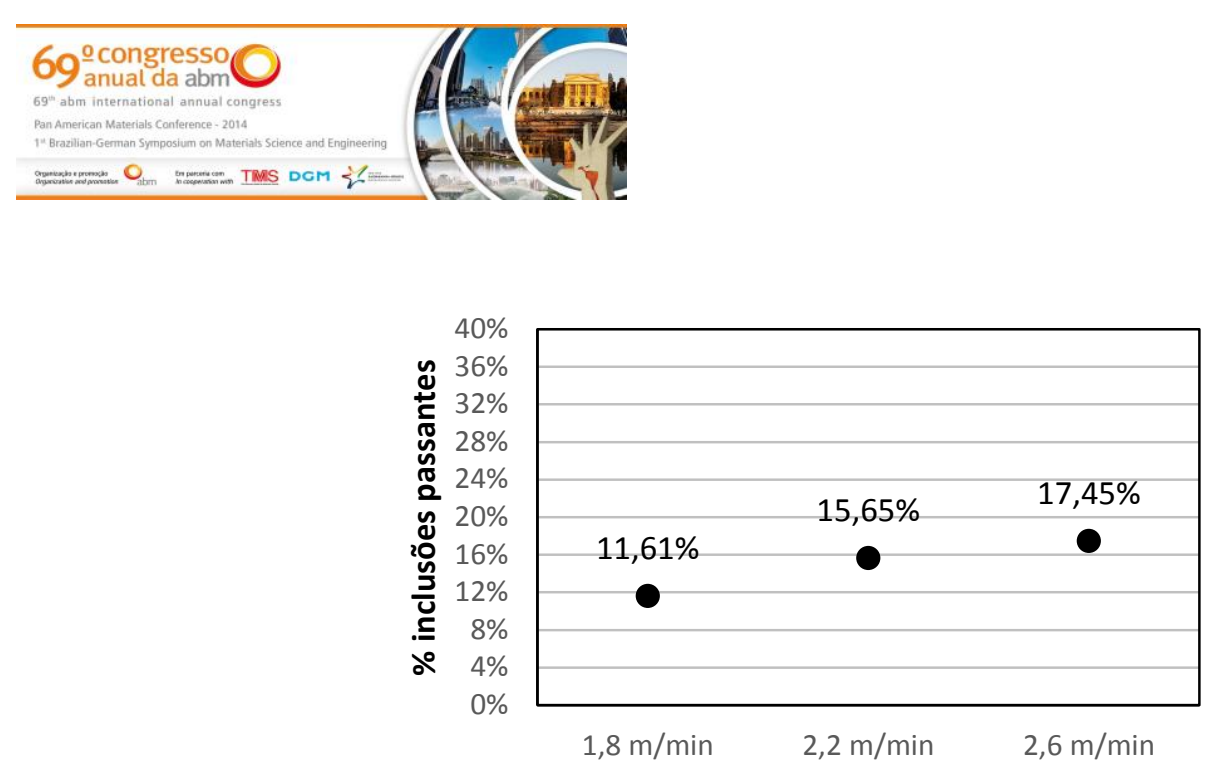

Velocidade de lingotamento

Figura 8. Porcentual de inclusões passantes em função da velocidade de lingotamento

A Figura 9 mostra que a presença do tubo longo influencia fortemente no percentual de inclusões passantes pelo veio (variação de 2,79 para 27,03\%). Atribui-se a esse contraste nos resultados entre a utilização de jato aberto (sem tubo longo) e o jato fechado (com tubo longo) a dois fenômenos presentes no distribuidor, ambos gerados pelo choque do jato de entrada com a interface ar/água e vistos no ensaio de linhas de fluxo. O primeiro deles é a quebra da velocidade do escoamento gerada por esse choque do jato de entrada, fenômeno comentado nas figuras 3 e 4 . 0 segundo fenômeno é o arraste de bolhas gerado quando o jato atravessa a interface ar/água (Figura 10). Lembrando que as bolhas capturam as inclusões e beneficiam a remoção das inclusões do aço [9].



Figura 9. Porcentual de inclusões passantes em função da presença do tubo longo

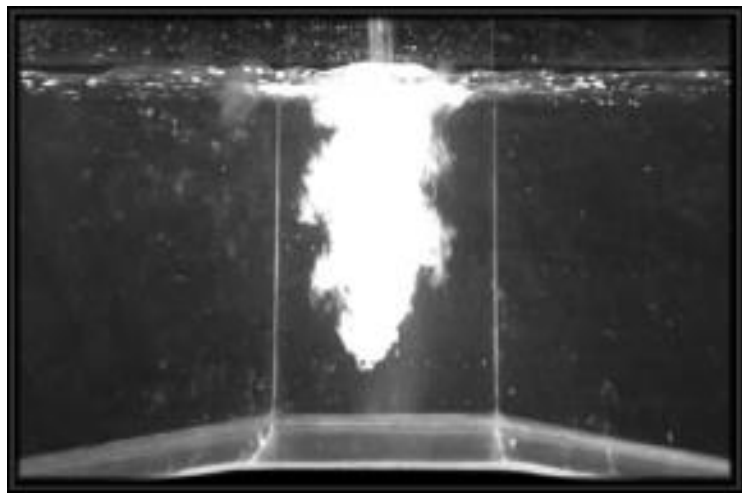

Figura 10. Arraste de bolhas para o seio do banho metálico gerado na ausência do tubo longo

\footnotetext{
* Contribuição técnica ao 69 Congresso Anual da ABM - Internacional e ao $14^{\circ}$ ENEMET - Encontro Nacional de Estudantes de Engenharia Metalúrgica, de Materiais e de Minas, 21 a 25 de julho de 2014, São Paulo, SP, Brasil.
} 


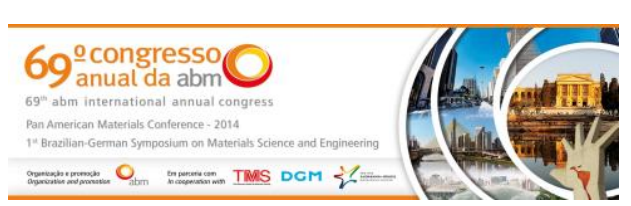

Contudo, quando é abordada a presença do tubo longo é importante ressaltar que a ausência deste pode aumentar a formação de inclusões via reoxidação do banho metálico. Logo, deve-se lembrar de que os ensaios que envolvem a ausência do tubo longo não consideram inclusões geradas pela reoxidação via exposição do aço líquido à atmosfera. Tal quantificação poderia alterar os percentuais de inclusões passantes nesses ensaios.

A Figura 11 mostra que o efeito principal da presença de barreiras diminui a passagem de inclusões para o molde de 16,16 para $13,65 \%$. Esse comportamento mostra que as barreiras podem ser utilizadas para melhorar o escoamento no distribuidor, como eliminar curto-circuito. Lembrando que esse resultado é uma média entre as configurações com tubo longo e sem tubo longo com o objetivo de avaliar o comportamento médio das barreiras em todas as configurações testadas. Logo, permanece a interpretação de que para o caso sem tubo longo as barreiras são maléficas e para o caso com tubo longo as barreiras são benéficas (ver comentário da Figura 7).

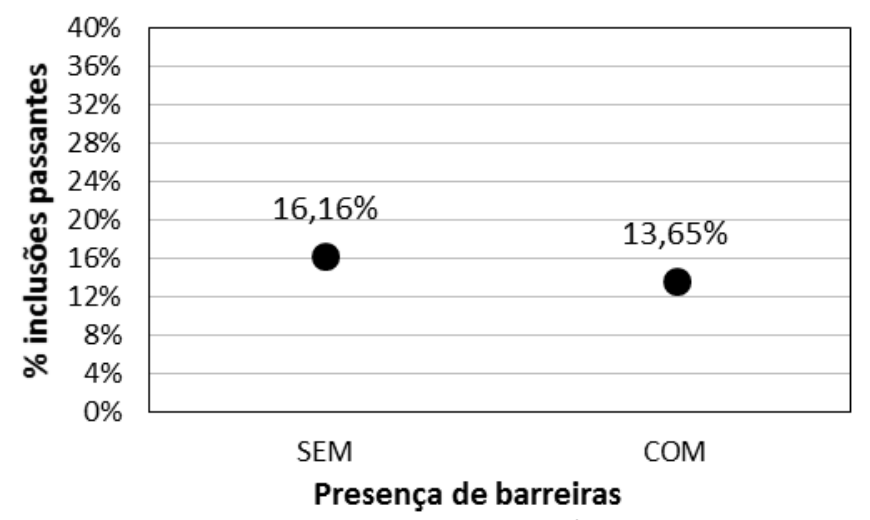

Figura 11. Porcentual de inclusões passantes em função da presença das barreiras

Na Figura 12 é mostrado que o aumento da velocidade de lingotamento tem maior intensidade sobre as configurações com tubo longo, dado que há um maior aumento no porcentual de inclusões passantes de 20,55 para $32,18 \%$ (com tubo longo) comparado ao aumento de 2,68 para $2,73 \%$ das configurações sem tubo longo. Isso pode ser entendido devido a captura das inclusões realizadas pelas bolhas presentes nas configurações sem tubo longo (Figura 10) que podem diminuir o efeito da velocidade de lingotamento sobre as inclusões passantes pelo veio.

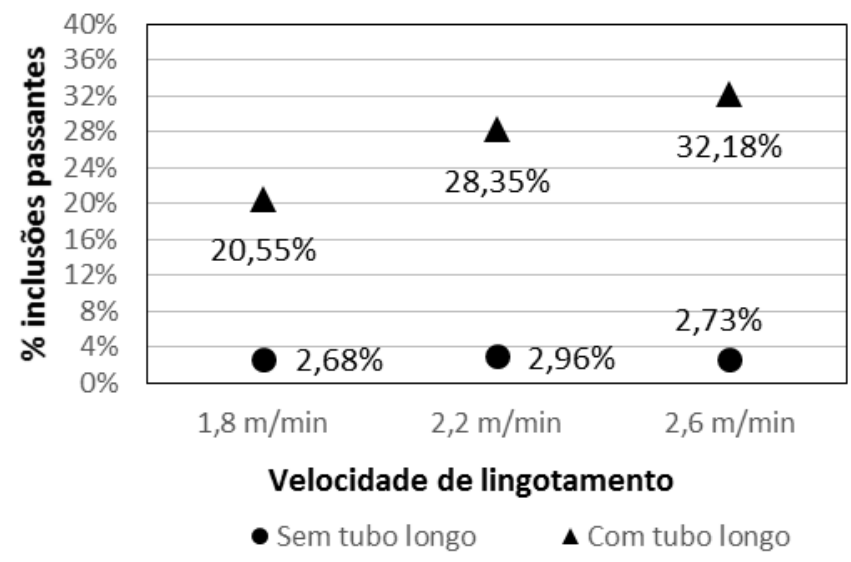

Figura 12. Porcentual de inclusões passantes em função da combinação entre a presença do tubo longo e a velocidade de lingotamento

* Contribuição técnica ao 69 Congresso Anual da ABM - Internacional e ao 14ํㅡㄹ ENET - Encontro Nacional de Estudantes de Engenharia Metalúrgica, de Materiais e de Minas, 21 a 25 de julho de 2014, São Paulo, SP, Brasil. 


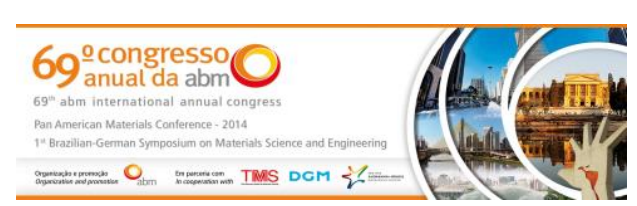

A configuração com tubo longo e com barreiras é a melhor configuração sob o ponto de vista do ensaio de linhas de fluxo e comparado à configuração com tubo longo e sem barreiras, esta configuração diminui as inclusões passantes pelo veio de 29,76 para $24,29 \%$.

$\mathrm{Na}$ média dos ensaios e configurações estudadas, o aumento da velocidade de lingotamento provoca um aumento no porcentual de inclusões de 11,61 para $17,45 \%$, a utilização do tubo longo também apresenta efeito significativo de aumento na quantidade de inclusões no modelo físico estudado (2,79\% sem tubo longo para $27,03 \%$ com tubo longo) e o emprego das barreiras diminui significativamente a quantidade de inclusões passantes no distribuidor utilizado $(16,16 \%$ sem barreiras para $13,65 \%$ com barreiras).

\section{Agradecimentos}

Aos nossos orientadores, Antônio Cezar Faria Vilela; Magda Beatriz Galant François e Felipe das Dôres Machado, por nos auxiliar a compor este trabalho, ajudando em todas as dificuldades do grupo. À Fundação Luiz Englert pelas bolsas de iniciação cientifica concedidas.

\section{REFERÊNCIAS}

1 Rogler JP, Heaslip LJ, Mehrvar M. Physical Modelling of Inclusion Removal in a Tundish by gas bubbling. Canadian Metallurgical Quarterly, 2005, 44(3): 357-68.

2 Zhang L. et al. Fluid Flow and Inclusion Removal in Continuos Casting Tundish. Mettalurgical and material transactions B, 2000; 31B: 253-266.

3 Qiang Y. et al. Water Modeling of Swirling Flo Tundish for steeel Continuos Casting. Journal of iron and steel research, International, 2009; 16: 17-22.

4 Joo S, Guthrie RIL. Inclusion behavior and heat-transfer phenomena in steelmaking tundish operations: Part I. Aqueous Modeling. Metallurgical transactions, 1993; 24: 75565.

5 Sahai Y, Emi T. Tundish technology for clean steel production. World Scientific Publishing Company, 2007.

6 Machado FD. Modelagem física de remoção de inclusões em distribuidor de lingotamento contínuo de tarugos. Dissertação de mestrado a ser defendida em 24 de março de 2014. Porto Alegre: Universidade Federal do Rio Grande do Sul, 2014.

7 Mazumdar D, Guthrie ILR. The physical and mathematical modeling of continuous casting tundish systems. ISIJ Intern., 1999; 39(6): 524-47.

8 François BMG, Machado, D. F. E Vilela, A. C. F. Remoção de inclusões em modelos físicos de distribuidores de lingotamento contínuo de aços: uma revisão bibliográfica. XXXXIV Seminário de Aciaria, Associação Brasileira de Metalurgia e Materiais - ABM, Araxá, MG, Brasil, 2013.

9 Rogler JP, Heaslip LJ, Mehrvar M. Inclusion removal in a tundish by gas bubbling. Canadian Metallurgical Quarterly, 2004; 43: 407-16.

\footnotetext{
* Contribuição técnica ao $69^{\circ}$ Congresso Anual da ABM - Internacional e ao 14ํㅡㄹ ENEMET - Encontro Nacional de Estudantes de Engenharia Metalúrgica, de Materiais e de Minas, 21 a 25 de julho de 2014, São Paulo, SP, Brasil.
} 